DOI: 10.12731/wsd-2018-1-136-150

УДК 616-093/-098

\title{
МОРФОМЕТРИЧЕСКАЯ И ЦВЕТОВАЯ ХАРАКТЕРИСТИКА КИСЛОТОУСТОЙЧИВЫХ МИКОБАКТЕРИЙ КАК ОБЬЕКТОВ НА ЦИФРОВЫХ ИЗОБРАЖЕНИЯХ МОКРОТЫ, ОКРАШЕННОЙ ПО МЕТОДУ ЦИЛЯ-НИЛЬСЕНА
}

Наркевич А.Н., Виноградов К.А.

Цель. Изучение морфометрических и цветовых характеристик кислотоустойчивых микобактерий как объектов на иифровых изображениях мокроты, окрашенной по методу Циля-Нильсена.

Материалы и методы. Использовались данные о 6708 кислотоустойчивых микобактериях, выделенных на ииифровых изображениях микроскопических препаратов мокроты, окраменной по методу Циля-Нильсена. Анализ объектов производился по 97 ияветовым и морфометрическим признакам.

Результаты. Наименее важными параметрами кислотоустойчивых микобактерий, как объектов на иифровых изображениях, для параметризации являются: минимальное значение $R$, максимальное значение $G$, минимальное значение $B$, максимальное значение $S$ и максимальное значение $V$, а наиболее информативными параметрами с точки зрения параметризачии объектов являются: максимальное значение $B$, среднее значение $V$, минимальное значение $H$, минимальное значение $V$ и максимальное значение $Y$ и среднее значение Н. В работе также представлены методики определения морфометрических характеристик кислотоустойчивых микобактерий при изменении разрешения цифровых изображений, на которых осуществляется поиск таких объектов, и изменении увеличения, при котором осуществляется микроскопия окрашенных образиов мокроты.

Заключение. Учет полученных в результате исследования морфометрических и цветовых характеристик позволит осуществлять разработку алгоритмов сегментации ицифровых микроскопических изображений мокроты, окрашенной по методу Циля-Нильсена, а также математических моделей распознавания объектов на данных изображениях. 
Ключевые слова: морфометрия; математическое моделирование; кислотоустойчивые микобактерии; бактериоскопическая диагностика; ијифровые изображения.

\title{
MORPHOMETRIC AND COLOR FEATURES ACID-FAST BACILLI AS OBJECTS ON DIGITAL IMAGES OF SPUTUM STAINED BY THE METHOD OF ZIEHL-NIELSEN
}

\author{
Narkevich A.N., Vinogradov K.A.
}

Background. Study of morphometric and color characteristics of acid-resistant mycobacteria as objects in digital images of sputum stained by the method of Ziehl-Nielsen.

Materials and methods. Used data on 6,708 acid-resistant mycobacteria isolated from digital images of microscopic preparations of sputum stained by the Ziehl-Nielsen method were used. The analysis of objects was made on 97 color and morphometric signs.

Result. The least important parameters of acid-resistant mycobacteria, as objects in digital images, for the parameterization of the minimum value of $R$, the maximum value of $G$, the minimum value of $B$, the maximum value of $S$ and the maximum value of $V$, and the most informative parameters in terms of the parameterization of objects are the maximum value of $B$, the average value of $V$, the minimum value of $H$, the minimum value of $V$ and the maximum value of $Y$ and the average value of $H$. The paper also presents methods for determining the morphometric characteristics of acid-resistant mycobacteria when changing the resolution of digital images, which search for such objects, and change the magnification in which the microscopy of colored sputum samples.

Conclusion. The use of the morphometric and color characteristics obtained as a result of the study will allow the development of algorithms for segmentation of digital microscopic images of sputum stained by the tsilya-Nielsen method, as well as mathematical models for recognition of objects in these images.

Keywords: morphometric; mathematical modeling; acid-fast mycobacteria; bacterioscopic diagnostics; digital images. 
Повсеместная компьютеризация приводит к тому, что решение большого числа медицинских задач перекладывается на «плечи» математических моделей и компьютерных технологий $[1,2,3,4]$. Широкое применение в медицинских исследованиях получили методы логистического регрессионного анализа $[5,6,7,8]$, дискриминантного анализа $[9,10,11,12]$, искусственных нейронных сетей $[13,14,15,16]$.

Одной из задач, с которой сталкиваются исследователи в области разработки таких математических моделей, является определение параметров, которые будут использоваться при описании интересующих объектов и которые будут использоваться непосредственно в математических моделях $[17,18]$.

Целью исследования является изучение морфометрических и цветовых характеристик кислотоустойчивых микобактерий как объектов на цифровых изображениях мокроты, окрашенной по методу Циля-Нильсена.

\section{Материалы и методы исследования}

Материалом исследования явились 6708 объектов КУМ, выделенных с использованием одного из разработанного нами алгоритма, на цифровых изображениях микроскопических препаратов мокроты, окрашенной по методу Циля-Нильсена [19], полученных с помощью тринокулярного микроскопа Микромед 1 вар. 3-20 при увеличении 10×60 с установленной цифровой камерой ТоupCam UCMOS01300KPA с разрешением 0,3 MP. Каждое изображение имело разрешение 572 х 422 пикселей.

Проверка на нормальность распределения всех параметров осуществлялась с использованием критерия Шапиро-Уилка.

Описание объектов производилось по 97 цветовым и морфометрическим признакам [20]. К цветовым признакам (всего - 24 признака) относились: среднее арифметическое, минимальное и максимальное значения объектов, составляющих R, G, B, H, S, V цветовых схем RGB и HSV. Также рассчитывались среднее арифметическое, минимальное и максимальное значения яркости (Y) пикселей объектов, что связано с довольно частым использованием для анализа цифровых изображений их представления в оттенках серого, а не в цветном виде. Измерение цветовых признаков осуществлялось в безразмерных единицах, однако интервал возможных значений отличался в зависимости от цветовой схемы. Так, цвета объектов в цветовой схеме RGB и в оттенках серого измерялись в интервале от 0 до 255, параметры, отражающие Н в цветовой схеме HSV, измерялись в интервале от 0 до 359, а параметры, характеризующие S и V, измерялись в интервале от 0 до 100 . 
Помимо представленных выше цветовых характеристик объектов использовались: индекс зеленого (Green Index - GI), индекс зеленых листьев (Green Leaf Index - GLI) и красно-зеленый индекс растительности (RedGreen vegetation index - RGVI), которые довольно часто используются в задачах описания объектов на цифровых изображениях. Их использование обусловлено специфическим восприятием цветов человеком [17].

Индекс зеленого рассчитывался по следующей формуле:

$$
G I=\frac{G}{R+G+B} .
$$

Индекс зеленых листьев:

$$
G L I=\frac{2 G-R-B}{2 G+R+B}
$$

Красно-зеленый индекс растительности:

$$
R G V I=\frac{G-R}{G+R}
$$

Индекс зеленого может принимать значения от 0 до 1 , а индекс зеленых листьев и красно-зеленый индекс растительности могут принимать значения от -1 до 1 .

К морфометрическим признакам (всего - 73 признака) относились: попиксельная площадь объектов, радиальные размеры объектов (всего - 72 размера). Радиальные размеры - длинна отрезка от центра объекта до его края, построенного под различным углом относительно вертикальной оси объекта с шагом $5^{\circ}$. Так первый отрезок построен под углом $5^{\circ}$, второй $-10^{\circ}$, третий $15^{\circ}$ и т.д. Морфометрические признаки объектов измерялись в пикселях.

Для описания цветовых характеристик объектов рассчитывались: среднее арифметическое (М), среднеквадратическое отклонение (б), коэффициент вариации (V), максимальное (Max) и минимальное (Min) значения, размах вариации $(\mathrm{R})$, коэффициент осцилляции $\left(\mathrm{V}_{\mathrm{R}}\right)$, медиана $(\mathrm{Me})$, первый $\left(\mathrm{Q}_{1}\right)$ и третий $\left(\mathrm{Q}_{3}\right)$ квартили, межквартильный размах $\left(\mathrm{R}_{\mathrm{Q}}\right)$ и коэффициент относительной квартильной вариации $\left(\mathrm{V}_{\mathrm{Q}}\right)$.

Для радиальных размеров объектов рассчитывались: среднее арифметическое, среднеквадратическое отклонение и максимальное значение размера по всем объектам.

\section{Результаты исследования и их обсуждение}

Описательная характеристика попискельной площади и цветовых характеристик изучаемых объектов КУМ представлены в таблице 1. 
Необходимо отметить, что наименее важными параметрами объектов КУМ для параметризации являются: минимальное значение $\mathrm{R}$, максимальное значение $\mathrm{G}$, минимальное значение $\mathrm{B}$, максимальное значение $\mathrm{S}$ и максимальное значение V ввиду того, что между объектами КУМ по данным параметрам отсутствует какая-либо вариация.

Наиболее информативными параметрами с точки зрения параметризации объектов на основе нормального распределения являются: максимальное значение $\mathrm{B}$, среднее значение $\mathrm{V}$, минимальное значение $\mathrm{H}$, минимальное значение $\mathrm{V}$ и максимальное значение $\mathrm{Y}$ ввиду их минимальной вариации у объектов КУМ. Данные параметры имеют наименьшие значения коэффициентов вариации и осцилляции.

Таблицуа 1.

Описательные параметры попиксельной площади и цветовых характеристик объектов КУМ

\begin{tabular}{|c|c|c|c|c|c|c|c|c|c|c|c|c|}
\hline Параметр & M & $\sigma$ & $\mathrm{V}$ & $\operatorname{Max}$ & Min & $\mathrm{R}$ & $\mathrm{V}_{\mathrm{R}}$ & $\mathrm{Me}$ & $\mathrm{Q}_{1}$ & $\mathrm{Q}_{3}$ & $\mathrm{R}_{\mathrm{Q}}$ & $\mathrm{V}_{\mathrm{Q}}$ \\
\hline Попиксельная площадь & 20,88 & 12,32 & 58,99 & 162,00 & 4,00 & 158,00 & 756,70 & 18,00 & 12,00 & 26,00 & 14,00 & 77,78 \\
\hline Минимальное значение R & 0,00 & 0,00 & - & 0,00 & 0,00 & 0,00 & - & 0,00 & 0,00 & 0,00 & 0,00 & - \\
\hline Среднее значение $\mathrm{R}$ & 122,72 & 36,27 & 29,55 & 240,00 & 1,00 & 239,00 & 194,75 & 124,00 & 100,00 & 146,00 & 46,00 & 37,10 \\
\hline Максимальное значение R & 166,98 & 38,54 & 23,08 & 255,00 & 5,00 & 250,00 & 149,72 & 168,00 & 145,00 & 193,00 & 48,00 & 28,57 \\
\hline Минимальное значение G & 145,22 & 25,46 & 17,53 & 206,00 & 25,00 & 181,00 & 124,64 & 150,00 & 133,00 & 162,00 & 29,00 & 19,33 \\
\hline Среднее значение G & 176,33 & 21,78 & 12,35 & 225,00 & 73,00 & 152,00 & 86,20 & 179,00 & 165,00 & 191,00 & 26,00 & 14,53 \\
\hline Максимальное значение G & 255,00 & 0,00 & - & 255,00 & 255,00 & 0,00 & - & 255,00 & 255,00 & 255,00 & 0,00 & - \\
\hline Минимальное значение В & 0,00 & 0,00 & - & 0,00 & 0,00 & 0,00 & - & 0,00 & 0,00 & 0,00 & 0,00 & - \\
\hline Среднее значение В & 176,94 & 22,67 & 12,81 & 233,00 & 74,00 & 159,00 & 89,86 & 180,00 & 165,00 & 193,00 & 28,00 & 15,56 \\
\hline Максимальное значение В & 228,24 & 12,85 & 5,63 & 255,00 & 178,00 & 77,00 & 33,74 & 230,00 & 221,00 & 238,00 & 17,00 & 7,39 \\
\hline Минимальное значение Н & 119,18 & 9,28 & 7,79 & 120,00 & 0,00 & 120,00 & 100,69 & 120,00 & 120,00 & 120,00 & 0,00 & - \\
\hline Среднее значение Н & 235,93 & 26,27 & 11,13 & 353,00 & 30,00 & 323,00 & 136,91 & 230,00 & 218,00 & 249,00 & 31,00 & 13,48 \\
\hline Максимальное значение Н & 255,48 & 29,35 & 11,49 & 359,00 & 203,00 & 156,00 & 61,06 & 249,00 & 233,00 & 272,00 & 39,00 & 15,66 \\
\hline Минимальное значение S & 27,51 & 14,31 & 52,02 & 98,00 & 1,00 & 97,00 & 352,60 & 25,00 & 17,00 & 35,00 & 18,00 & 72,00 \\
\hline Среднее значение S & 47,25 & 13,59 & 28,76 & 99,00 & 17,00 & 82,00 & 173,54 & 46,00 & 37,00 & 56,00 & 19,00 & 41,30 \\
\hline Максимальное значение S & 100,00 & 0,00 & - & 100,00 & 100,00 & 0,00 & - & 100,00 & 100,00 & 100,00 & 0,00 & - \\
\hline Минимальное значение V & 81,81 & 7,01 & 8,57 & 100,00 & 54,00 & 46,00 & 56,23 & 82,00 & 77,00 & 87,00 & 10,00 & 12,20 \\
\hline Среднее значение V & 88,33 & 5,23 & 5,92 & 100,00 & 67,00 & 33,00 & 37,36 & 89,00 & 85,00 & 92,00 & 7,00 & 7,87 \\
\hline Максимальное значение V & 100,00 & 0,00 & - & 100,00 & 100,00 & 0,00 & - & 100,00 & 100,00 & 100,00 & 0,00 & - \\
\hline Минимальное значение Y & 140,92 & 16,86 & 11,96 & 150,00 & 46,00 & 104,00 & 73,80 & 150,00 & 139,00 & 150,00 & 11,00 & 7,33 \\
\hline Среднее значение Y & 160,23 & 19,65 & 12,26 & 214,00 & 68,00 & 146,00 & 91,12 & 162,00 & 151,00 & 173,00 & 22,00 & 13,58 \\
\hline Максимальное значение Y & 175,86 & 17,96 & 10,21 & 230,00 & 150,00 & 80,00 & 45,49 & 175,00 & 162,00 & 188,00 & 26,00 & 14,86 \\
\hline Среднее значение GI & 0,37 & 0,05 & 13,51 & 0,61 & 0,23 & 0,38 & 102,70 & 0,37 & 0,34 & 0,40 & 0,06 & 16,22 \\
\hline Среднее значение GLI & 0,08 & 0,10 & 125,00 & 0,52 & $-0,26$ & 0,78 & 975,00 & 0,07 & 0,02 & 0,14 & 0,12 & 171,43 \\
\hline Среднее значение RGVI & 0,20 & 0,14 & 70,00 & 0,98 & $-0,25$ & 1,23 & 615,00 & 0,18 & 0,09 & 0,27 & 0,18 & 100,00 \\
\hline
\end{tabular}


С позиции распределения, отличающегося от нормального с точки зрения параметризации объектов КУМ, наиболее информативными являются: минимальное значение Y, максимальное значение В, среднее значение V, минимальное значение $\mathrm{V}$ и среднее значение $\mathrm{H}$, так как данные параметры имеют наименьшие значения коэффициента относительной квартильной вариации. В связи с тем, что все анализируемые параметры не подчиняются закону нормального распределения, то более предпочтительным набором параметров для описания объектов КУМ являются отобранные на основании коэффициента относительной квартильной вариации.

Средние значения и среднеквадратическое отклонение радиальных размеров объектов КУМ представлены на рисунке 1. Как видно из данных приведенных на рисунке 1, средние значения радиальных размеров объектов КУМ напоминают форму «палочки», в виде которой КУМ описываются с позиции микробиологии.

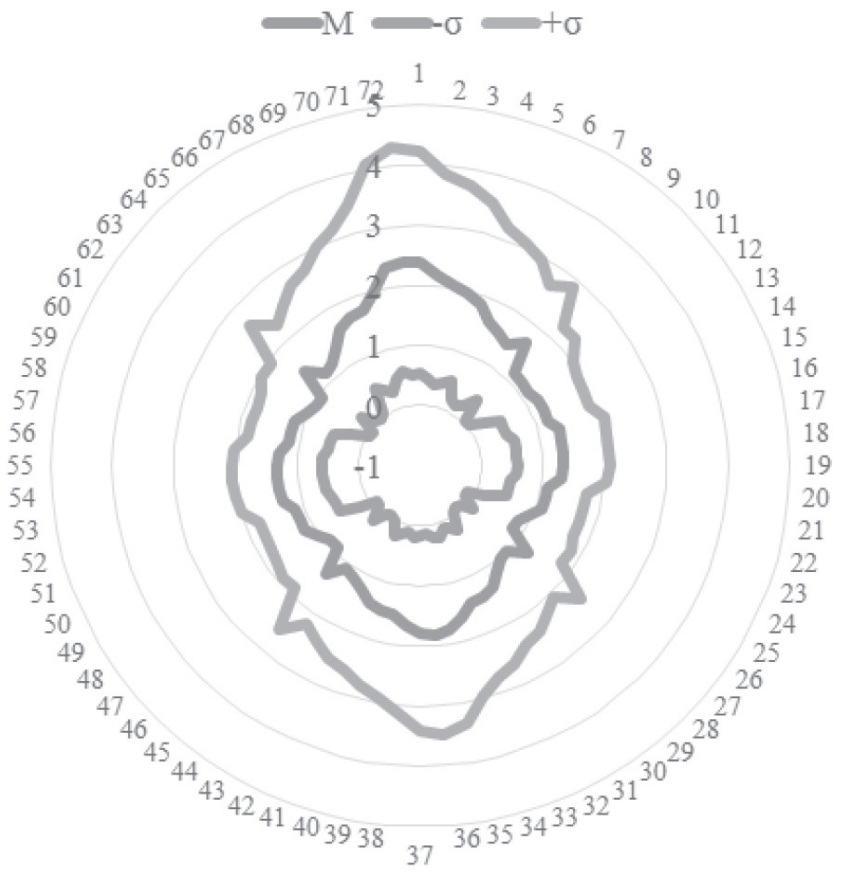

Рис. 1. Среднее арифметическое и стандартное отклонение радиальных размеров кислотоустойчивых микобактерий 
Необходимо отметить, что наибольшую ценность для описания имеют размеры описывающие боковые размеры объектов КУМ. Наименьшие значения коэффициентов вариации, осцилляции и относительной квартильной вариации имеют радиальные размеры от 13 до 23 и от 49 до 59, что соответствует углам от $65^{\circ}$ до $115^{\circ}$ и от $245^{\circ}$ до $295^{\circ}$.

Максимальные радиальные размеры изучаемых объектов КУМ представлены на рисунке 2.

Так как изучение морфометрических характеристик объектов КУМ осуществлялось на цифровых изображениях, имеющих определенное разрешение (572x422 пикселей), и полученные при определенном увеличении (10×60), то представленные морфометрические параметры являются непостоянными при изменении разрешения и увеличения. Однако, при изменении разрешения цифрового изображения или увеличения, при котором делается цифровая фотосъемка, отсутствует необходимость повторного изучения морфометрических характеристик объектов КУМ. Данные параметры могут быть получены расчетным путем на основании полученных нами результатов.

Для определения радиальных размеров при изменении разрешения цифровой фотосъемки без изменения соотношения размеров сторон изображения можно воспользоваться следующей формулой:

$$
r_{i}=\sqrt{\frac{x * y}{241384}} * r_{0, i},
$$

где $r_{i}$ - итоговая длина радиального размера объекта под углом $i, x$ - число пикселей изображения по горизонтали, у - число пикселей изображения по вертикали, 241384 - число пикселей на изучаемых нами изображениях $\left(572 * 422=241384\right.$ пикселя), $r_{0, i}$ - длина радиального размера объекта под углом і на изучаемых нами изображениях.

Также может быть определена и попиксельная площадь объектов при изменении разрешения цифровой фотосъемки:

$$
S=\frac{x * y}{241384} * S_{0}
$$

где $S$ - итоговая попиксельная площадь объекта, $x$ - число пикселей изображения по горизонтали, у - число пикселей изображения по вертикали, 241384 - число пикселей на изучаемых нами изображениях, $S_{0}$ - попиксельная площадь объекта на изучаемых нами изображениях.

Необходимо отметить, что формула определения площади объектов при изменении разрешения изображения может применяться независимо от того меняется ли соотношение сторон изображения или нет. 


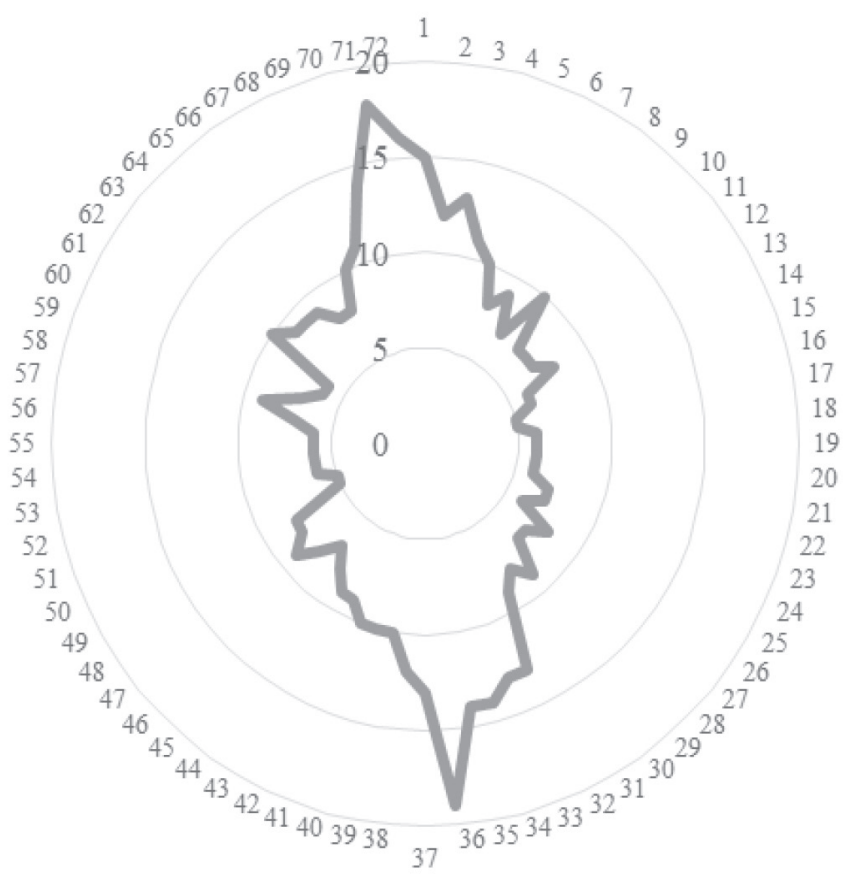

Рис. 2. Максимальные значения радиальных размеров кислотоустойчивых микобактерий

Для определения радиального размера при изменении разрешения цифровой фотосъемки с изменением соотношения размеров сторон изображения для начала необходимо определить относительные координаты края объекта $\left(x_{0} ; y_{0}\right)$, к которому построен отрезок данного размера:

$$
x_{0}=\left\{\begin{array}{l}
r_{0, i} * \cos (i-90), \text { при } i \geq 90 \\
r_{0, i} * \cos (i+360-90), \text { при } i<90
\end{array}\right.
$$

где $x_{0}$ - координата по оси $x, r_{0, i}$ длина радиального размера объекта под углом $i$ на изучаемых нами изображениях.

$$
y_{0}=\left\{\begin{array}{l}
r_{0, i} * \sin (i-90), \text { при } i \geq 90 \\
r_{0, i} * \sin (i+360-90), \text { при } i<90
\end{array}\right.
$$

где $y_{0}$ - координата по оси у, $r_{0, i}$ - длина радиального размера объекта под углом $i$ на изучаемых нами изображениях. 
Далее необходимо пересчитать значения новых относительных координат края объекта, к которому будет построен отрезок искомого радиального размера после изменения разрешения изображения:

$$
x_{\text {new }}=x_{0} * \frac{\text { width }}{572},
$$

где $x_{\text {new }}$ - новая координата по оси $x, x_{0}$ - координата по оси $x$, width - ширина изображения в пикселях, 572 - ширина изучаемых нами изображений в пикселях.

$$
y_{\text {new }}=y_{0} * \frac{h e i g h t}{422},
$$

где $y_{\text {new }}$ - новая координата по оси $y, y_{0}$ - координата по оси $y$, height - высота изображения в пикселях, 422 - высота изучаемых нами изображений в пикселях.

И далее, используя новые координаты края объекта, можно рассчитать длину отрезка искомого радиального размера после изменения разрешения изображения:

$$
r_{i}=\sqrt{x_{\text {new }}^{2}+y_{\text {new }}^{2}},
$$

где $r_{i}$ - итоговая длина радиального размера объекта под углом $i, x_{\text {new }}-$ новая координата по оси $x, y_{\text {new }}$ - новая координата по оси $y$.

При изменении увеличения, при котором осуществляется цифровая фотосъемка, соотношение сторон изображения не изменяется, в связи с чем определение радиальных размеров объектов может осуществляться с использованием данной формулы:

$$
r_{i}=\sqrt{\frac{z}{600}} * r_{0, i}
$$

где $r_{i}$ - итоговая длина радиального размера объекта под углом $i, Z-$ увеличение, 600 - увеличение при получении изучаемых нами изображений $(10 * 60=600), r_{0, i}-$ длина радиального размера объекта под углом $i$ на изучаемых нами изображениях.

Попиксельная площадь объектов определяется:

$$
S=\frac{Z}{600} * S_{0},
$$

где $S$ - итоговая попиксельная площадь объекта, $Z$ - увеличение, 600 - увеличение при получении изучаемых нами изображений, $S_{0}-$ попиксельная площадь объекта на изучаемых нами изображениях. 
Необходимо отметить, что цветовые характеристики объектов не подвержены изменению при изменении разрешения изображения или увеличение при микроскопии.

\section{Заключение}

Таким образом, нами рассмотрены морфометрические и цветовые характеристики кислотоустойчивых микобактерий как объектов на цифровых изображениях мокроты, окрашенной по методу Циля-Нильсена, а также рассмотрены методики определения данных характеристик при изменении разрешения цифровых изображений, на которых осуществляется поиск таких объектов, и изменении увеличения, при котором осуществляется микроскопия окрашенных образцов мокроты.

Учет полученных в результате исследования морфометрических и цветовых характеристик позволит осуществлять разработку алгоритмов сегментации цифровых микроскопических изображений мокроты, окрашенной по методу Циля-Нильсена, а также математических моделей распознавания объектов на данных изображениях.

\section{Список литературь}

1. Kaplan A., Lock E.F. Prediction with dimension reduction of multiple molecular data sources for patient survival // Cancer informatics. 2017. № 16. e1176935117718517.

2. Automated seizure detection using limited-channel EEG and non-linear dimension reduction / Birjandtalab J., Baran Pouyan M., Cogan D., Nourani M., Harvey J. // Computers in biology and medicine. 2017. № 82, pp. $49-58$.

3. Грицинская В.Л., Москаленко О.Л. Использование компьютерных технологий при проведении диспансеризации детского населения Республики Тыва // В мире научных открытий. 2017. № 2. С. 158-167.

4. Supervised nonlinear dimension reduction of functional magnetic resonance imaging data using Sliced Inverse Regression / Tu Y., Tan A., Fu Z., Sam Hung Y., Hu L., Zhang Zh. // Conference proceedings: 37th Annual International Conference of the IEEE Engineering in Medicine and Biology Society. Milan, 2015, pp. 2641-2645.

5. Стрижов В.В., Мотренко А.П. Многоклассовая логистическая регрессия для прогноза вероятности наступления инфаркта // Известия Тульского государственного университета. Естественные науки. 2012. № 1. C. $153-162$. 
6. Гарганеева Н.П., Леонов В.П. Логистическая регрессия в анализе связи артериальной гипертонии и психических расстройств // Сибирский медицинский журнал. 2001. Т. 16. № 3-4. С. 42-48.

7. Микшина В.С., Павлов С.И. Использование логистической регрессии при выборе способа кардиоплегии // Вестник Томского государственного университета. Управление, вычислительная техника и информатика. 2017. № 39. C. 49-56.

8. Андосова Л.Д., Конторщикова К.Н., Шахова К.А. Многофакторный регрессионный анализ в прогнозе развития цервикальных поражений инфекционного генеза // Медицинский альманах. 2017. № 2 (47). С. 111-113.

9. Возможности электрокардиографии в диагностике гипертрофии миокарда левого желудочка / Богомолов С.Н., Солнцев В.Н., Куликов А.Н., Кучмин А.Н. // Вестник Российской военно-медицинской академии. 2016. № 3 (55). С. 51-56.

10. Системы оценки контролируемости бронхиальной астмы / Безруков Н.С., Еремин Е.Л., Колосов В.П., Перельман Ю.М., Хижняк Ю.Ю. // Информатика и системы управления. 2009. № 4 (22). С. 159-163.

11. Быстрицкая Т.С., Штель Н.Н., Лысяк Д.С. Прогнозирование плацентарной недостаточности у беременных с нарушением становления менструальной функции в пубертатном периоде // Бюллетень физиологии и патологии дыхания. 2011. № 42. С. 55-59.

12. Прозорова А.В., Приходько А.Г. Построение прогнозной модели прогрессирующего течения хронической обструктивной болезни легких // Информатика и системы управления. 2008. № 2 (16). С. 168-169.

13. Филист С.А., Шаталова О.В., Ефремов М.А. Гибридная нейронная сеть с макрослоями для медицинских приложений // Нейрокомпьютеры: разработка, применение. 2014. № 6. С. 35-69.

14. Барский А.Б., Дмитриев А.А., Барская О.А. Медицинские информационно-справочные системы на логических нейронных сетях // Информационные технологии. 2010. № S1. С. 1-32.

15. Петров С.Б., Шешунов И.В. Оценка эффективности применения искусственных нейронных сетей в медико-экологических исследованиях // Фундаментальные исследования. 2013. № 9-6. С. 1098-1101.

16. Муха Ю.П., Скворцов М.Г., Дружинин Д.Г. Метрологическое описание нейронных сетей // Биомедицинские технологии и радиоэлектроника. 2002. № 4. C. $20-23$.

17. Feature selection method based on high-resolution remote sensing images and the effect of sensitive features on classification accuracy / Zhou Y., Zhang R., Wang S., Wang F. // Sensors. 2018. № 18. 2013. 
18. Extraction and parametrization of grain boundary networks in glacier ice, using a dedicated method of automatic image analysis / Binder T., Garbe C.S., Wagenbach D., Freitag J., Kipfstuhl S. // Journal of Microscopy. 2013. № 2, pp. 130-141.

19. Сегментация микроскопических изображений мокроты, окрашенной по методу Циля-Нильсена, с использованием вейвлет-преобразования Mexican Hat / Наркевич А.Н., Виноградов К.А., Корецкая Н.М., Соболева B.O. // Acta Biomedica Scientifica. 2017. T. 2. № 5. C. 141-146.

20. Наркевич А.Н., Виноградов К.А., Корецкая Н.М. Параметризация объектов на цифровых микроскопических изображениях мокроты, окрашенной по методу Циля-Нильсена // Сибирское медицинское обозрение. 2017. № 5 (107). C. 53-59.

\section{References}

1. Kaplan A., Lock E.F. Prediction with dimension reduction of multiple molecular data sources for patient survival. Cancer informatics, 2017, № 16, e1176935117718517.

2. Birjandtalab J., Baran Pouyan M., Cogan D., Nourani M., Harvey J. Automated seizure detection using limited-channel EEG and non-linear dimension reduction. Computers in biology and medicine, 2017, № 82, pp. 49-58.

3. Gritsinskaya V.L., Moskalenko O.L. Ispol'zovanie komp'yuternykh tekhnologiy pri provedenii dispanserizatsii detskogo naseleniya Respubliki Tyva [The use of computer technologies at carrying out of prophylactic medical examination of children of the Republic of Tuva]. V mire nauchnykh otkrytiy [In the world of scientific discoveries], 2017, № 2, pp. 158-167.

4. Tu Y., Tan A., Fu Z., Sam Hung Y., Hu L., Zhang Zh. Supervised nonlinear dimension reduction of functional magnetic resonance imaging data using Sliced Inverse Regression. Conference proceedings: 37th Annual International Conference of the IEEE Engineering in Medicine and Biology Society, Milan, 2015, pp. 2641-2645.

5. Strizhov V.V., Motrenko A.P. Mnogoklassovaya logisticheskaya regressiya dlya prognoza veroyatnosti nastupleniya infarkta [Multi-class logistic regression to predict the probability of a heart attack]. Izvestiya Tul'skogo gosudarstvennogo universiteta. Estestvennye nauki [Proceedings of the Tula state University. More natural science], 2012, № 1, pp. 153-162.

6. Garganeeva N.P., Leonov V.P. Logisticheskaya regressiya $v$ analize svyazi arterial'noy gipertonii i psikhicheskikh rasstroystv [Logistic regression in the analysis of the relationship between hypertension and mental upset]. Sibir- 
skiy meditsinskiy zhurnal [Siberian medical journal], 2001, Vol. 16, № 3-4, pp. 42-48.

7. Mikshina V.S., Pavlov S.I. Ispol'zovanie logisticheskoy regressii pri vybore sposoba kardioplegii [The use of logistic regression in choosing a method of cardioplegia]. Vestnik Tomskogo gosudarstvennogo universiteta. Upravlenie, vychislitel'naya tekhnika i informatika [Vestnik of Tomsk state University. Management, computer engineering and computer science], 2017, № 39, pp. 49-56.

8. Andosova L.D., Kontorshchikova K.N., Shakhova K.A. Mnogofaktornyy regressionnyy analiz $\mathrm{v}$ prognoze razvitiya tservikal'nykh porazheniy infektsionnogo geneza [Multivariate regression analysis in prediction of cervical porazheniy infectious Genesis]. Meditsinskiy al'manakh [Medical almanac], 2017, № 2, pp. 111-113.

9. Bogomolov S.N., Solntsev V.N., Kulikov A.N., Kuchmin A.N. Vozmozhnosti elektrokardiografii $v$ diagnostike gipertrofii miokarda levogo zheludochka [Possibilities of electrocardiography in the diagnosis of left ventricular hypertrophy]. Vestnik Rossiyskoy voenno-meditsinskoy akademii [Bulletin of the Russian military medical Academy], 2016, № 3, pp. 51-56.

10. Bezrukov N.S., Eremin E.L., Kolosov V.P., Perel'man Yu.M., Khizhnyak Yu.Yu. Sistemy otsenki kontroliruemosti bronkhial'noy astmy [Evaluation system accountability bronchial asthma]. Informatika i sistemy upravleniya [Informatics and control systems], 2009, № 4, pp. 159-163.

11. Bystritskaya T.S., Shtel' N.N., Lysyak D.S. Prognozirovanie platsentarnoy nedostatochnosti u beremennykh s narusheniem stanovleniya menstrual'noy funktsii $\mathrm{v}$ pubertatnom periode [Prediction placentary insufficiency in pregnant women with impaired formation menstrual function in the pubertal period]. Byulleten' fiziologii i patologii dykhaniya [Bulletin of physiology and pathology of respiration], 2011, № 42, pp. 55-59.

12. Prozorova A.V., Prikhod'ko A.G. Postroenie prognoznoy modeli progressiruyushchego techeniya khronicheskoy obstruktivnoy bolezni legkikh [Constructing a predictive model of progressive course of chronic obstructive pulmonary disease]. Informatika i sistemy upravleniya [Informatics and control systems], 2008, № 2, pp. 168-169.

13. Filist S.A., Shatalova O.V., Efremov M.A. Gibridnaya neyronnaya set's makrosloyami dlya meditsinskikh prilozheniy [Hybrid neural network macro slope for medical applications]. Neyrokomp'yutery: razrabotka, primenenie [Neurocomputers: development, application], 2014, № 6, pp. 35-69.

14. Barskiy A.B., Dmitpiev A.A., Bapskaya O.A. Meditsinskie informatsionno-spravochnye sistemy na logicheskikh neyronnykh setyakh [Medical in- 
formation and referral system for Boolean neural networks]. Informatsionnye tekhnologii [Information technologies], 2010, № 1, pp. 1-32.

15. Petrov S.B., Sheshunov I.V. Otsenka effektivnosti primeneniya iskusstvennykh neyronnykh setey v mediko-ekologicheskikh issledovaniyakh [Evaluation of the effectiveness of the use of artificial neural networks in medical and environmental research]. Fundamental'nye issledovaniya [Fundamental study], 2013, № 9-6, pp. 1098-1101.

16. Mukha Yu.P., Skvortsov M.G., Druzhinin D.G. Metrologicheskoe opisanie neyronnykh setey [Metrological description of neural networks]. Biomeditsinskie tekhnologii i radioelektronika [Biomedical technologies and radioelectronics], 2002, № 4, pp. 20-23.

17. Zhou Y., Zhang R., Wang S., Wang F. Feature selection method based on high-resolution remote sensing images and the effect of sensitive features on classification accuracy. Sensors. 2018. № 18. 2013.

18. Binder T., Garbe C.S., Wagenbach D., Freitag J., Kipfstuhl S. Extraction and parametrization of grain boundary networks in glacier ice, using a dedicated method of automatic image analysis. Journal of Microscopy. 2013. № 2, pp. 130-141.

19. Narkevich A.N., Vinogradov K.A., Koretskaya N.M., Soboleva V.O. Segmentatsiya mikroskopicheskikh izobrazheniy mokroty, okrashennoy po metodu Tsilya-Nil'sena, s ispol'zovaniem veyvlet-preobrazovaniya Mexican Hat [Segmentation of microscopic images of sputum stained by the method of Ziehl-Nielsen, using wavelet transform Mexican Hat]. Acta Biomedica Scientifica [Acta Biomedica Scientifica], 2017, Vol. 2, № 5, pp. 141-146.

20. Narkevich A.N., Vinogradov K.A., Koretskaya N.M. Parametrizatsiya ob"ektov na tsifrovykh mikroskopicheskikh izobrazheniyakh mokroty, okrashennoy po metodu Tsilya-Nil'sena [Parameterization of objects on digital microscopic images of sputum stained by the method of Ziehl-Nielsen]. Sibirskoe meditsinskoe obozrenie [Siberian medical review], 2017, № 5, pp. 53-59.

\section{ДАННЫЕ ОБ АВТОРАХ}

Наркевич Артем Николаевич, заведующий научно-исследовательской лабораторией медицинской кибернетики и управления в здравоохранении, кандидат медицинских наук

Красноярский государственный медицинский университет имени профессора В.Ф. Войно-Ясенеикого

ул. Партизана Железняка, 1, г. Красноярск, 660022, Российская Федерачия

narkevichart@gmail.com 
Виноградов Константин Анатольевич, заведующий кафедрой медицинской кибернетики и информатики, доктор медицинских наук, профессор

Красноярский государственный медицинский университет имени профессора В.Ф. Войно-Ясенеикого

ул. Партизана Железняка, 1, г. Красноярск, 660022, Российская Федераичи

vinogradov16@yandex.ru

\section{DATA ABOUT THE AUTHORS}

Narkevich Artem Nikolaevich, Head of Research Laboratory of Medical Cybernetics and Management in Health Care, Candidate of Medical Sciences

Krasnoyarsk State Medical University

1, Partisana Zheleznyaka Str., Krasnoyarsk, 660022, Russian Federation narkevichart@gmail.com

Vinogradov Konstantin Anatolevich, Head of the Department of Medical Cybernetics and Informatics, Doctor of Medical Sciences, Professor Krasnoyarsk State Medical University

1, Partisana Zheleznyaka Str., Krasnoyarsk, 660022, Russian Federation vinogradov16@yandex.ru 